\title{
MEGARA: a new generation optical spectrograph for GTC
}

A. Gil de Paz ${ }^{* a}$, J. Gallego $^{\mathrm{a}}$, E. Carrasco ${ }^{\mathrm{b}}$, J. Iglesias-Páramo ${ }^{\mathrm{c}}$, R. Cedazo ${ }^{\mathrm{d}}, \mathrm{J}^{\mathrm{M}}$ M. Vílchez ${ }^{\mathrm{c}}$, M.L. García Vargas $^{\mathrm{e}}$, X. Arrillaga ${ }^{\mathrm{f}}$, M. A. Carrera ${ }^{\mathrm{f}}$, A. Castillo-Morales ${ }^{\mathrm{a}}$, E. Castillo-Domínguez ${ }^{\mathrm{b}}$, M.C.

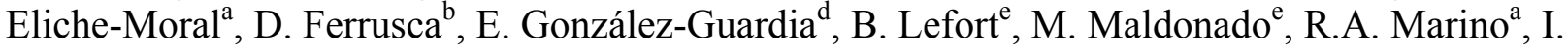
Martínez-Delgado $^{\mathrm{e}}$, I. Morales Durán ${ }^{\mathrm{c}}$, E. Mujica ${ }^{\mathrm{e}}$, G. Páez $^{\mathrm{g}}, \mathrm{S}_{\text {. Pascual }}^{\mathrm{a}}$, A. Pérez-Calpena ${ }^{\mathrm{e}}, \mathrm{A}$. Sánchez-Penim $^{\mathrm{a}}$, E. Sánchez-Blanco ${ }^{\mathrm{e}}$, S. Tulloch ${ }^{\mathrm{e}}$, M. Velázquez ${ }^{\mathrm{b}}$, J. Zamorano ${ }^{\mathrm{a}}$, A.L. Aguerri,i, D.

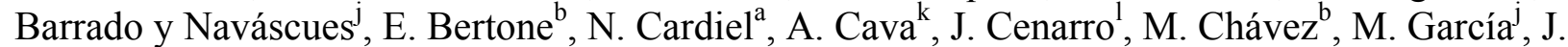
Guichard $^{\mathrm{b}}$, R. Gúzman ${ }^{\mathrm{m}}$, A. Herrero ${ }^{\mathrm{h}, \mathrm{i}}$, N. Huélamo ${ }^{\mathrm{j}}$, D. Hughes ${ }^{\mathrm{b}}$, J. Jiménez-Vicente ${ }^{\mathrm{n}}$, C. Kehrig ${ }^{\mathrm{c}}$, I. Márquez $^{c}, J_{\text {. Masegosa }}^{c}$, Y. D. Mayya ${ }^{b}, J_{\text {. Méndez-Abreu }}^{o}$, M. Molláp ${ }^{\text {, C. Muñoz-Tuñón }}{ }^{\text {h,i }}$, M. Peimbert $^{\mathrm{q}}$, P.G. Pérez-González ${ }^{\mathrm{a}}$, E. Pérez Montero ${ }^{\mathrm{c}}$, M. Rodríguez ${ }^{\mathrm{b}}$, J.M. Rodríguez-Espinosa ${ }^{\text {h,i }}$, L. Rodríguez-Merino $^{b}$, D. Rosa-González ${ }^{b}$, J. Sánchez-Almeida, ${ }^{\text {h,i }}$ C. Sánchez Contreras ${ }^{j}$, P. SánchezBlázquez $^{\mathrm{r}}$, F.M. Sánchez Moreno ${ }^{\mathrm{d}}$, S. F. Sánchez ${ }^{\mathrm{q}}$, A. Sarajedini ${ }^{\mathrm{m}}$, F. Serena ${ }^{\mathrm{d}}$, S. Silich ${ }^{\mathrm{b}}$, S. SimónDíaz $^{\text {h,i }}$, G. Tenorio-Tagle ${ }^{\mathrm{b}}$, E. Terlevich $^{\mathrm{b}}$, R. Terlevich $^{\mathrm{b}}, \mathrm{S}$. Torres-Peimbert ${ }^{\mathrm{q}}$, I. Trujillo ${ }^{\mathrm{h}, \mathrm{i}}$, Y. Tsamis, O. Vega ${ }^{\mathrm{b}}, \mathrm{V}$. Villar ${ }^{\mathrm{a}}$

${ }^{\mathrm{a} U n i v e r s i d a d ~ C o m p l u t e n s e ~ d e ~ M a d r i d ~(U C M, ~ S p a i n), ~}{ }^{\mathrm{b}}$ Instituto Nacional de Astrofísica, Óptica y Electrónica (INAOE, Mexico), ${ }^{c}$ Instituto de Astrofísica de Andalucía (IAA-CSIC, Spain),

${ }^{\mathrm{d}}$ Universidad Politécnica de Madrid (UPM, Spain), ${ }^{\mathrm{e}}$ FRACTAL SLNE (Spain), ${ }^{\mathrm{f}}$ AVS SL, ${ }^{\mathrm{g}}$ Centro de Investigaciones en Óptica (Mexico), ${ }^{\mathrm{h}}$ Instituto de Astrofísica de Canarias (Spain), ${ }^{\mathrm{i}}$ Universidad de

La Laguna (Spain), ${ }^{\mathrm{j}}$ Centro de Astrobiología (Spain), ${ }^{\mathrm{k}}$ University of Geneva $(\mathrm{CH}),{ }^{1}$ Centro de Estudios de Física del Cosmos de Aragón (Spain), ${ }^{\mathrm{m}}$ University of Florida (USA), ${ }^{\mathrm{n}}$ Universidad de Granada (Spain), ${ }^{\mathrm{o}}$ University of St. Andrews, ${ }^{\mathrm{p}}$ CIEMAT (Spain), ${ }^{\mathrm{q}}$ Universidad Autónoma de México (Mexico), ${ }^{\mathrm{r}}$ Universidad Autónoma de Madrid (Spain), ${ }^{\mathrm{s}}$ Open University (UK)

\begin{abstract}
MEGARA (Multi-Espectrógrafo en GTC de Alta Resolución para Astronomía) is an optical Integral-Field Unit (IFU) and Multi-Object Spectrograph (MOS) designed for the GTC 10.4m telescope in La Palma. MEGARA offers two IFU fiber bundles, one covering 12.5x11.3 $\operatorname{arcsec}^{2}$ with a spaxel size of $0.62 \operatorname{arcsec}$ (Large Compact Bundle; LCB) and another one covering 8.5x6.7 $\operatorname{arcsec}^{2}$ with a spaxel size of $0.42 \operatorname{arcsec}$ (Small Compact Bundle; SCB). The MEGARA MOS mode will allow observing up to 100 objects in a region of $3.5 \times 3.5 \mathrm{arcmin}^{2}$ around the two IFU bundles.

Both the LCB IFU and MOS capabilities of MEGARA will provide intermediate-to-high spectral resolutions $\left(\mathrm{R}_{\mathrm{FWHM}} \sim 6,000,12,000\right.$ and 18,700, respectively for the low-, mid- and high-resolution Volume Phase Holographic gratings) in the range 3650-9700 $\AA$. These values become $\mathrm{R}_{\mathrm{FWHM}} \sim 7,000,13,500$, and 21,500 when the SCB is used.

A mechanism placed at the pseudo-slit position allows exchanging the three observing modes and also acts as focusing mechanism. The spectrograph is a collimator-camera system that has a total of 11 VPHs simultaneously available (out of the $18 \mathrm{VPHs}$ designed and being built) that are placed in the pupil by means of a wheel and an insertion mechanism. The custom-made cryostat hosts an E2V231-84 4kx4k CCD.

The UCM (Spain) leads the MEGARA Consortium that also includes INAOE (Mexico), IAA-CSIC (Spain), and UPM (Spain). MEGARA is being developed under a contract between GRANTECAN and UCM. The detailed design, construction and AIV phases are now funded and the instrument should be delivered to GTC before the end of 2016.

Keywords: instrumentation: spectrographs, techniques: spectroscopic, galaxies: Local Group, spiral, kinematics and dynamics, ISM: kinematics and dynamics

*agil@ucm.es; phone +34 91394 5152; fax +34 91394 4635; http:/guaix.fis.ucm.es/megara/
\end{abstract}

Ground-based and Airborne Instrumentation for Astronomy V, edited by

Suzanne K. Ramsay, lan S. McLean, Hideki Takami, Proc. of SPIE Vol. 9147,

914700 - () 2014 SPIE · CCC code: 0277-786X/14/\$18 - doi: 10.1117/12.2047825

Proc. of SPIE Vol. 9147 914700-1 


\section{INTRODUCTION}

MEGARA (Multi-Espectrógrafo en GTC de Alta Resolución para Astronomía) is an intermediate-resolution optical Integral-Field Unit (IFU) and Multi-Object Spectrograph (MOS) that will be installed at the $10.4 \mathrm{~m}$ GTC telescope on early 2017. The project started as a response of a Consortium of institutions led by the Universidad Complutense de Madrid (UCM, Spain) that also includes the Instituto Nacional de Astrofísica, Óptica y Electrónica (INAOE, Mexico), the Instituto de Astrofísica de Andalucía (IAA-CSIC, Spain) and the Universidad Politécnica de Madrid (UPM, Spain) to the Announcement of Opportunity issued by the Spanish public company GRANTECAN S.A. back in 2009. After being selected in a competitive Conceptual Design Review in September 2010, the instrument successfully passed its Preliminary (March 2012) and Detailed Optical Design Reviews (May 2013). On May 2014 the UCM, as leader institution, finally signed the Full Detailed Design, Construction, and AIV contract with GRANTECAN S.A. for a target delivery date at GTC on December 2016. The Full Detailed Design is scheduled for June 2014. In this paper we summarize the main characteristics of the instrument and the status of the project as a whole.

Section 2 provides an overview of the instrument subsystems. In Section 3 we provide a summary of the scientific objectives that defined the instrument requirements as agreed by the MEGARA Science Team. Sections 4 through 10 describe these subsystems in further detail, including the MEGARA Control System (MCS hereafter). Finally, the system engineering and management plan of the project are provided in Section 10. We refer the reader to other publications in this volume $\mathrm{e}^{1,2,3,4,5,6,7}$ for further details.

\section{OVERVIEW}

The instrument consists of two units, one located at the Folded-Cass (FC) focus, where the two IFUs and the robotic positioners of the MOS are placed and the other one at the Nasmyth platform, where the MEGARA spectrograph is placed and kept static. The Folded-Cass unit and the spectrograph are connected by means of a $40 \mathrm{~m}$-long fiber link ${ }^{2}$.

MEGARA offers two IFU modes with two different bundles, one covering $12.5 \operatorname{arcsec} \times 11.3 \operatorname{arcsec}$ with a spaxel size of 0.62 arcsec (Large Compact Bundle; LCB hereafter, which makes use of $100 \mathrm{~m}$-core optical fibers) and another one covering 8.5 arcsec x 6.7 arcsec with a spaxel size of $0.42 \operatorname{arcsec}$ (Small Compact Bundle; SCB hereafter, with 70 mcore fibers). MEGARA also provides a MOS mode that will allow observing up to 100 objects in a region of 3.5 arcmin x 3.5 arcmin around the two IFU bundles. Eight of these bundles will be devoted to the determination of the sky during the observation with the LCB IFU, so only 92 of these positioners will be available for MOS observations and will be controlled by the MEGARA Control System. Each of the three optical-fiber bundles is connected to the MEGARA spectrograph by means of curved telecentric pseudo-slits. The three pseudo-slits are placed on top of an x-y mechanism that allows exchanging between the three instrument modes and, at the same time, focusing the spectrograph for each spectral setup.

The MEGARA spectrograph is fully-refractive optical system composed by a collimator with 5 lenses (including an aspheric singlet and two doublets) and a camera with 7 lenses (three singlets and two doublets). The disperser elements are Volume Phase Holographic (VPH) gratings (sandwiched between flat windows) that provide resolutions between $\mathrm{R}_{\mathrm{FWHM}} \sim 6,000$ (in the case of the low-resolution LR VPHs), 12,000 (for the mid-resolution MRs) and 18,700 (for the high-resolution HRs) in the case of the LCB and MOS modes. MR and HR VPHs they both incorporate sets of two prisms that change the incident angle on the corresponding VPH. The VPHs are inserted in the pupil by means of an insertion mechanism that extracts the VPH from a wheel where up to a total of 11 VPHs can be placed (out of the 6 LR + $10 \mathrm{MR}+2 \mathrm{HR}=18 \mathrm{VPHs}$ designed and being built). More details on the MEGARA spectrograph optical design, optomechanics and manufacturing process are given in this same volume ${ }^{1,4,6}$. The camera focuses the light onto an E2V23184 deep-depleted $4 \mathrm{kx} 4 \mathrm{k} 15-\mu \mathrm{m}$ pixels CCD that is located in a custom cryostat designed and being built by the INAOE ${ }^{5}$.

The MCS will be responsible for all mechanisms in MEGARA (focal-plane cover, Fiber-MOS robotic positioners, pseud-slit exchange and focusing, shutter, and VPH wheel and insertion mechanism) and for the Data Acquisition System (DAS hereafter) and will follow the GTC standards defined by GRANTECAN. More details on the MCS can be found in Section 9 and elsewhere ${ }^{8}$.

The MEGARA System Engineering provides the methodology for developing a complex system, like MEGARA, in a structured and orderly manner and, therefore, helps to ensure that the whole instrument is correctly developed from the beginning, minimizing risks and anticipating problems that may arise. Finally, as in other major project, MEGARA includes a detailed management plan that, within a series of managerial parameters, is intended to ensure a timely 
delivery and successful AIV (Assembly, Integration and Verification) of MEGARA at GTC while satisfying all instrument and functional requirements, including those imposed by GRANTECAN and those defined by the MEGARA Science Team. More details on the MEGARA system engineering and management plan are provided in Section 10 and in other publications in this volume $\mathrm{e}^{3,7}$.

\section{MEGARA SCIENCE}

The scientific interests of the MEGARA Science Team can be grouped in two categories, (1) the study of Galactic and extragalactic extended nebulae and (2) the study of numerous sources (or close to point sources) clustered in the sky with intermediate-to-high surface densities. Category (1) includes the study of Planetary Nebulae, nearby galaxies, and the high-redshift IGM and category (2) Galactic open stellar clusters, resolved stellar populations in Local Group galaxies, intermediate-redshift dwarf and starburst galaxies, and high-redshift cluster galaxies. The MEGARA Science Team encompasses researchers with a broad range of scientific interests belonging to institutions of all members of the GTC community (Spain, Mexico and UF). This guarantees that, as a facility instrument, MEGARA will also successfully serve to the interests of the entire astronomical communities of the GTC Consortium members (see Figure 1 for a graphical summary of the main subjects of interest of our Science Team).

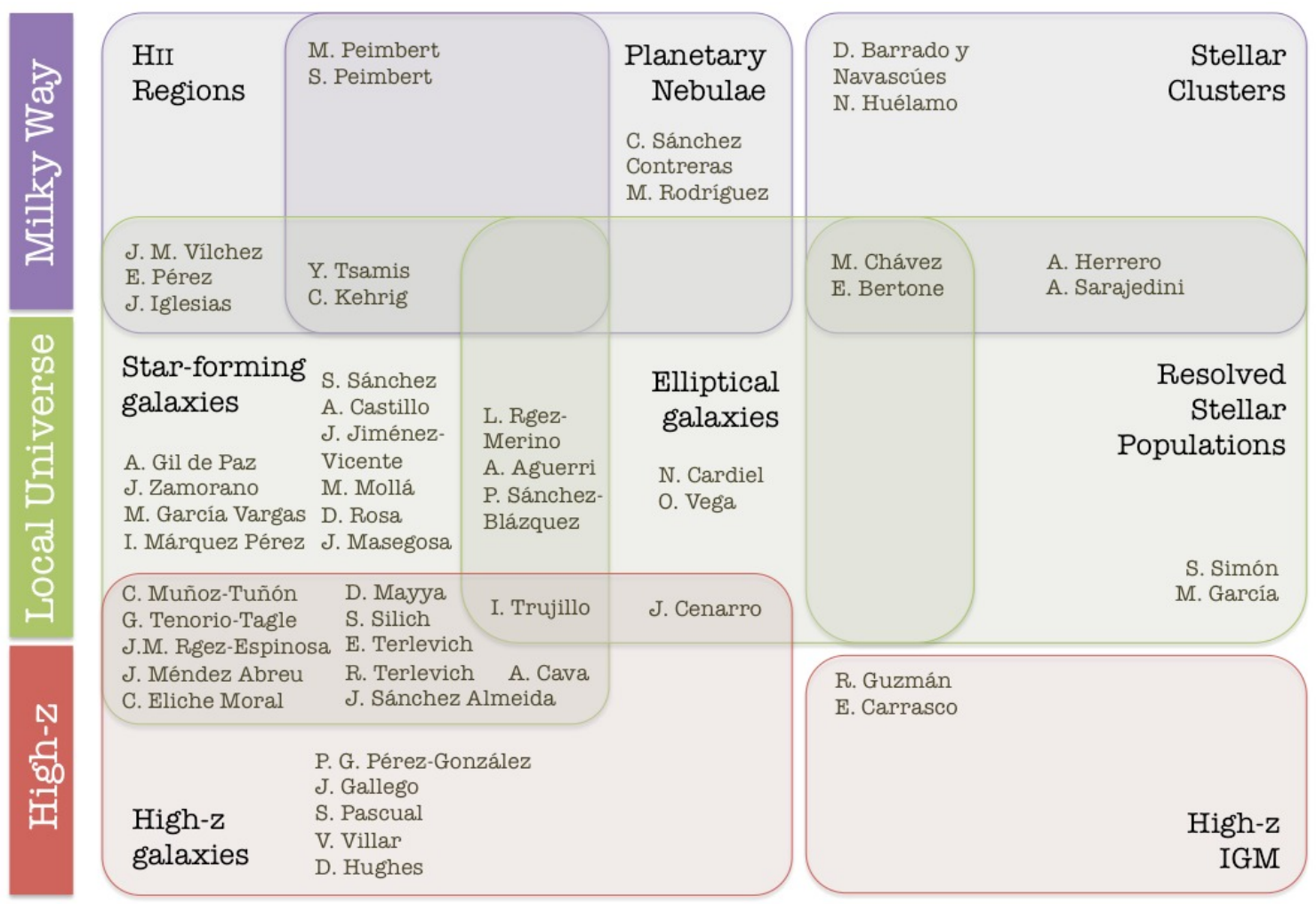

Figure 1. Areas of main scientific interest of the MEGARA Science Team members.

\subsection{IFU Science}

Among the study of astrophysical nebulae, our team has a strong interest in the understanding of the evolution of galaxy disks through the analysis of the velocity ellipsoids and the 2D spatial distribution of spectral indices and chemical abundances. This goal constitutes the core of the MEGARA Galaxy Disks Evolution Survey. This analysis is fundamental to disentangle the roles of the secular processes involved in the shaping the present-day properties of disks, i.e. in-situ star formation, stellar migration and satellite accretion and (minor-) merging among others. In addition to the study of the unresolved stellar populations in galaxy disks in the DAGAL/S4G sample ${ }^{9}$, this survey will also include the detailed 
single-star spectroscopic analysis of the Local Group galaxies M33 and IC 1613 using the MEGARA MOS mode. Other objectives that the MEGARA Galaxy Disks Evolution Survey dataset will allow also pursuing include the study of the interplay between the massive-star formation and the interstellar medium and the long-standing problem of the abundance discrepancies in HII regions and the potential identification and analysis of chemo-dynamical phenomena.

Other scientific topics that should make extensive use of the MEGARA IFUs are the study of Planetary Nebulae (PNe), the identification and analysis of arcs and highly magnified galaxies in clusters, and the mapping of the high-redshift intergalactic medium (IGM) through the blind search of the rest-frame UV resonant line emission in the blue part of the optical spectrum. Regarding the study of the PNe we aim at understanding, on one side, the nebular shaping process, which is believed to be driven by the interaction between collimated, fast (i.e. jet-like) winds ejected during the lateAGB or early post-AGB stages and the slow, largely spherical envelopes formed during the previous AGB phase as a result of a heavy mass-loss process. On the other side, we will use the superb efficiency of the MEGARA instrument combined with the large collecting area of GTC to analyze the faint metal recombination lines with an unprecedented combination of depth and spectral resolution so to determine the origin of the discrepancy between the element abundances derived from recombination and collisionally-excited lines and which lines actually provide the most reliable abundances and what method should thus be used to calculate the metallicity of the gas.

We aim to use the MEGARA IFUs to identify and analyze arcs and highly magnified galaxies in clusters. The faint continuum emission and relatively large projected size in the sky of some of these sources make necessary the use of an IFU in order to identify them and for spectroscopically measuring their redshifts, especially when they have been identified in the infrared ${ }^{10}$. Finally, the same blind search technique to be used in the high-magnification area of intermediate-redshift clusters could be used to search for redshifted emission of resonant lines in the UV rest-frame such as OVI1033 $\AA$, CIV1550 $\AA$, or Ly $\alpha$. This search would have been particular effective as part of parallel-observing programs back when the construction of two spectrographs was in the scope of the MEGARA project ${ }^{11,12}$. Nevertheless, we still consider this a feasible project especially when the search starts from the location of known bright Lyman Alpha Emitters (LAEs).

\subsection{MOS Science}

We plan to complement the study of nearby disks described above with MOS observations of resolved-stars in Local Group galaxies. Its inclination, location in the sky and relatively undisturbed morphology makes of the disk of M33 an ideal laboratory to analyze the 2D distribution of single-star abundances and kinematics that could provide further clues on the role of the different processes that shape the photometric, chemical and dynamical evolution of galaxy disks. MEGARA is optimally designed to study point sources with high number densities (such as RGB stars in Local Group galaxies or low-mass stars in Milky-Way clusters) as it allows reaching densities as high as 8 objects per $\operatorname{arcmin}^{2}$. This fact along with the short reconfiguration time of Fiber-MOS robotic positioners $(<1 \mathrm{~min}$; see Section 4$)$ makes of MEGARA a unique tool for the study of large numbers of sources in this kind of targets.

Indeed, we aim to study of properties of very low mass stars (and brown dwarfs) in young stellar associations from a holistic perspective. First, within the Gould Belt and, then, moving farther away, to the galactic anticenter and the Perseus Arm. The goal is to put star formation in a galactic perspective and to include the effects of both metallicity and environment. We will also use these observations to re-calibrate the activity-sensitive $\mathrm{Ca} \mathrm{H}+\mathrm{K}$ index with the stellar age for an extended sample of solar analogs in stellar open clusters in the context of the characterization of solar analogs candidates for the future search of Sun-Earth-like planetary systems.

There are also multiple interests within the MEGARA Science Team regarding the study of high-redshift galaxies: Intermediate-redshift Blue Compact Dwarf (BCD), clump-cluster, peas and starburst galaxies and high-redshift protoclusters. In order to address all these topics with maximum efficiency the capability for observing a relatively large number of objects within a small patch of the sky of the MEGARA MOS is a big advantage especially when carrying out studies on cosmological fields that aim to reach the spectroscopic limit.

\section{FOLDED-CASSEGRAIN SUBSYSTEMS}

The MEGARA Folded-Cassegrain (FC) subsystems include all components that collect and conduct the light from the focal plane to the spectrograph entrance. Those elements are (a) a Field Lens to correct from lack of telecentricity providing a telecentric focal plane for the microlens arrays, (b) the focal-plane cover that allows obtaining very low cross-talk observations with half the field-of-view (FoV) and multiplexing of the MEGARA default mode, (c) the 
microlenses that change the focal number of the telescope allowing a good coupling with fibers, (d) the fiber bundles, (e) the Fiber MOS that allows to position 92 minibundles in the focal plane, (f) the interface plate that supports the LCB and SBC IFUs in the central area of the focal plane, (g) the FC Rotator Adapter that provides the interface with the GTC FC rotator and (h) the interface between fibers, pseudo-slit plates where fibers are arranged and the Spectrograph entrance.

Figure 2 below shows different views of the FC subsystems and the adapter to the GTC FC rotator.

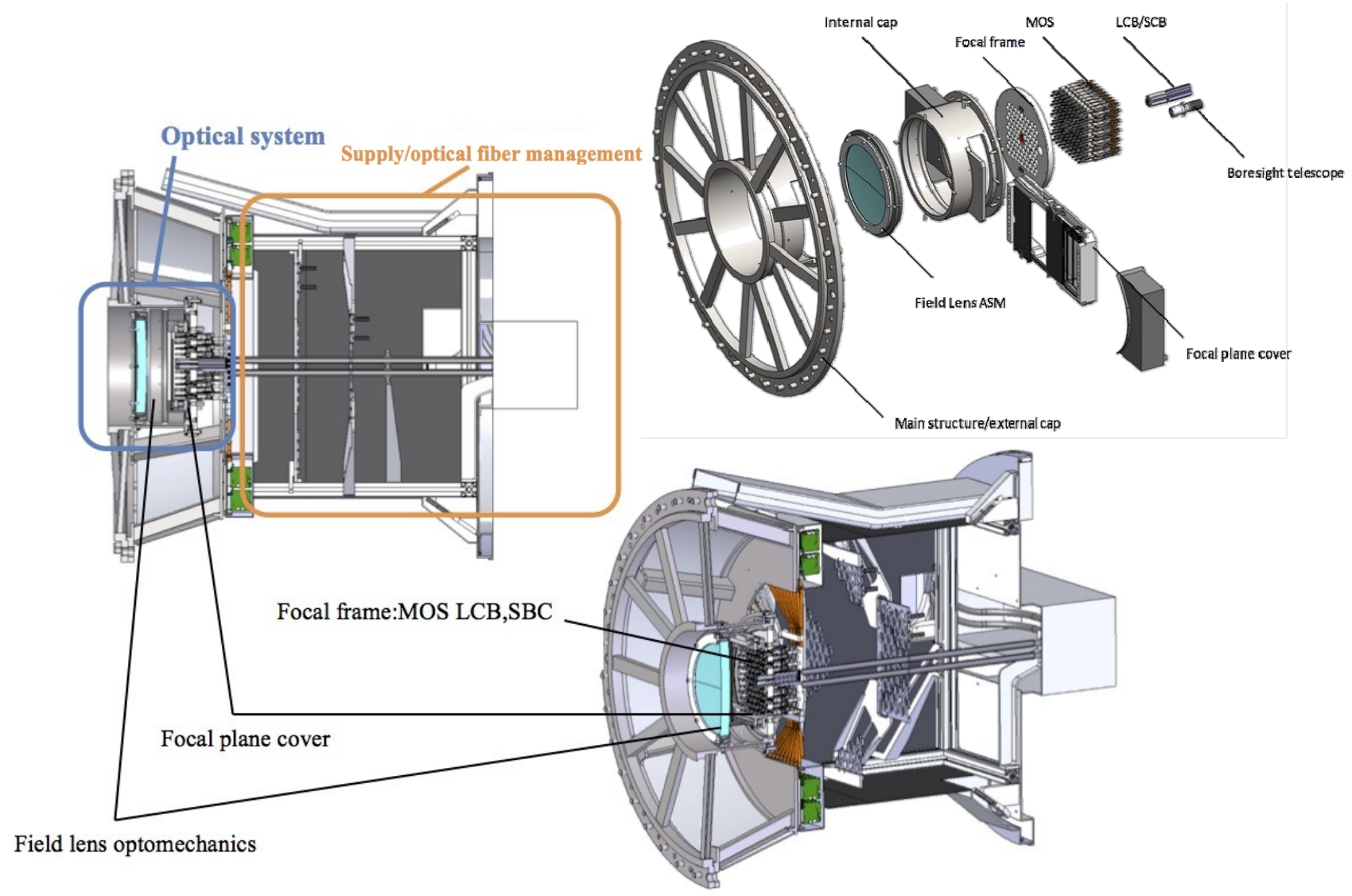

Figure 2. Folded-Cass adapter (left and bottom) and optical components exploded view (top-right).

Following the light path we first find the field lens (see top-right panel of Figure 2). This field lens has been designed to provide a telecentric field for MEGARA as the GTC telescope has the aperture at the secondary mirror. This allows that the opto-mechanical axes of all the fiber bundles will be parallel among them. Thus, the positioners move on a flat surface (the focal plane) with their opto-mechanical axis perpendicular to this surface and the two IFUs are also placed flat parallel. A small field curvature remains but it is below 0.1 arcsec in the whole FoV.

After the field lens we have the telescope focal plane where different microlens arrays adapt the telescope F\# from $\mathrm{f} / 17$ to $\mathrm{f} / 3$ to minimize focal ratio degradation (FRD) effects during the beam transport in the fibers and provide a telescope pupil image on the $100 \mu \mathrm{m}$ fiber core $(70 \mu \mathrm{m}$ in the case of the SCB) for stray light control. The MEGARA Instrument is composed of three modes, two IFUs and the Multi-Object Spectrograph (MOS) mode, that correspond to the three fiber bundles available: the Large Compact Bundle (LCB), the Small Compact Bundle (SCB) and the Dispersed Bundle or simply the Fiber MOS (see Figure 3). Each of these modes has a microlens array associated. In the case of the LCB the square-shaped lens array is composed of 567 lenslets, the SCB array includes 511 lenslets and the Fiber-MOS has 100 lens arrays (only 92 will have their fibers mounted on the MOS pseudo-slit; the other 8 will be mounted on the LCB pseudo-slit; see below) with 7 hexagonally-shaped and packed lenslets (these lenslets are identical to those of the LCB). 
Each LCB/MOS lenslet is a fused-silica plano-aspherical lens made of $\mathrm{ROC}=0.844 \mathrm{~mm}, \mathrm{c}=-0.9797$ and aperture of $0.511 \mathrm{~mm}$, while the SCB lenslets have a $\mathrm{ROC}=0.563 \mathrm{~mm}, \mathrm{c}=-0.927$ and an aperture of $0.346 \mathrm{~mm}$.

The lenslets that constitute the Large Compact Bundle (LCB) array are arranged on a square area of $12.5 \operatorname{arcsec} \times 11.3$ arcsec near the optical axis of the instrument, the lenslets that constitute the Small Compact Bundle (SCB) array are distributed in a square image area of. $8.5 \operatorname{arcsec} \times 6.7 \operatorname{arcsec}$, whose center is offset $\sim 19 \operatorname{arcsec}$ from the center of the LCB. The 92 7-lenslets arrays of the Fiber MOS can be positioned anywhere in the central 3.5 arcmin x 3.5 arcmin around the two IFU bundles thanks to the positioner robots (or simply positioners).

Note that a total of 8 robotic positioners (orange hexagons in Figure 3) located in the outer edge of the instrument FoV are kept fixed in order to be used for measuring the sky background simultaneously with the observations with the LCB. The layout of the hexagonally-packed (and shaped) microlens arrays of the LCB and SCB IFUs is also shown in this figure.

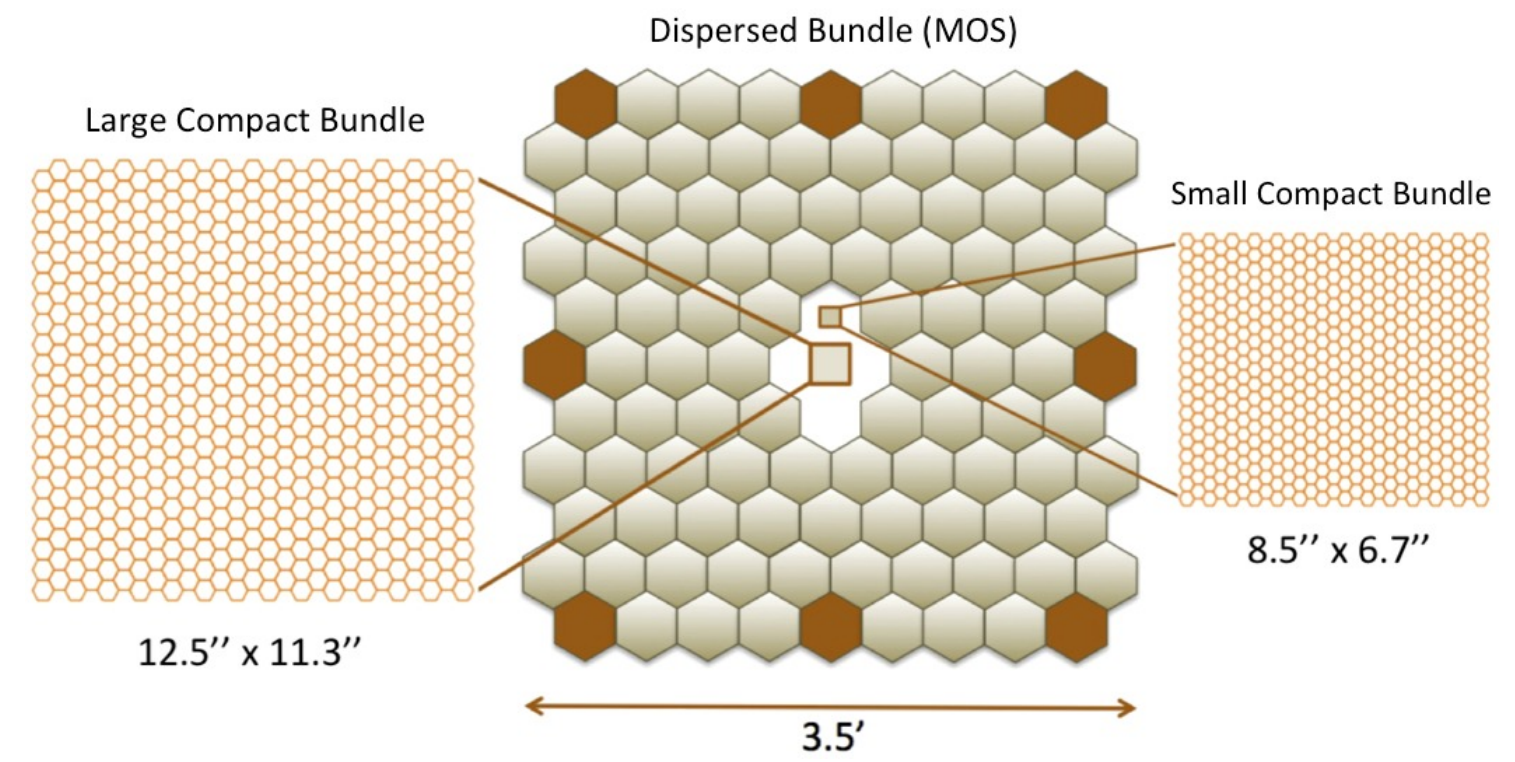

Figure 3. Layout of the LCB, SCB and MOS modes of MEGARA.

The GTC pupil image is formed at the back surface of each array and is overfilling the fiber core in order to provide an illumination condition as similar as possible to the one given by the Instrument Calibration Module (ICM) and to minimize the impact of either mounting or assembly errors between fibers and microlenses on the flux homogeneity in between fibers (maximum tolerance is set to $10 \mu \mathrm{m}$ for the LCB and MOS and $8 \mu \mathrm{m}$ for the SCB at the pupil image).

Each of the 100 microlens arrays of the MOS mode is hold by a robotic positioner (by means of a button) that moves independently of the rest allowing reconfiguring the entire focal plane in less than one minute of time. The positioning of the fiber minibundle is performed by combining the interpolation of two rotations, which allows covering a circle with a radius of $11.605 \mathrm{~mm}$ from the center of the positioner (this circle reaches the corners of the hexagon with an $\mathrm{E} / \mathrm{C}$ of $20.1 \mathrm{~mm}$ ). The fixing of the positioner button to the positioner, which shall be strong and repetitive, has been designed considering that it should be possible to disassembly it from its back in order to facilitate the maintenance of the mechanical and electronics parts (see Figure 4). The length of the feeding and the signal cables and the fibers shall be also defined taking into account the need to assembly and disassembly each positioner independently. The prototype of one of the positioners was completed during the Preliminary Design phase and was presented during the PDR on March 2012. This unit is shown in Figure 4 (before the optical fiber bundle was attached on December 2011). 

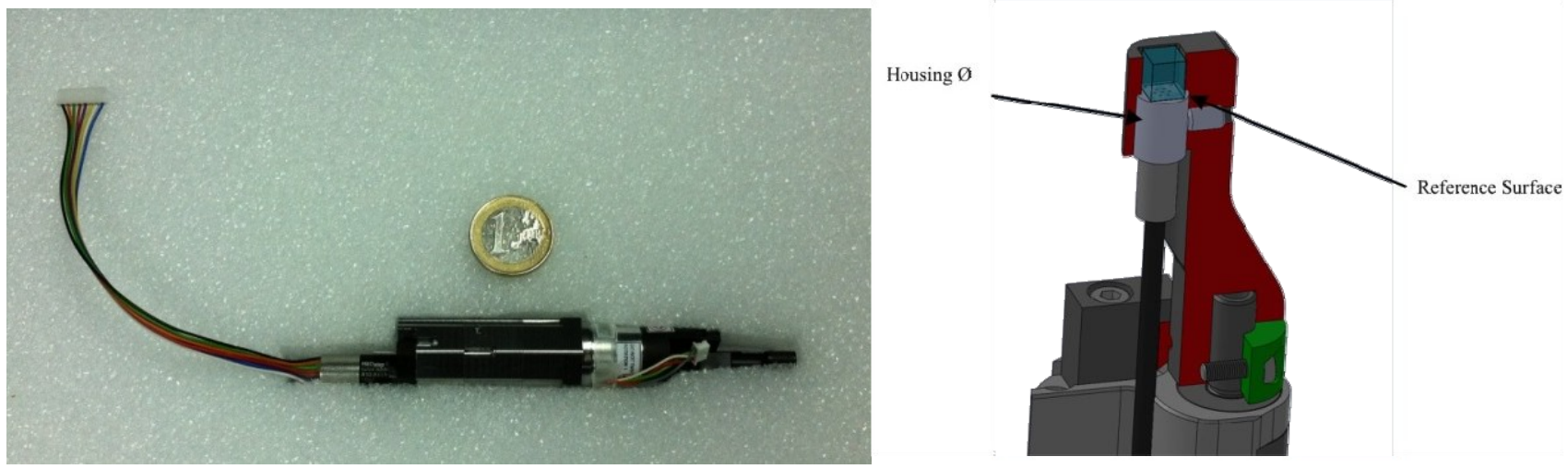

Figure 4. Left: Fiber MOS positioner prototype (December 2011). Right: Positioner button detailed design.

\section{FIBER LINK}

The fiber cables transport the light from the focal plane to the spectrographs at the pseudo-slit position. The characteristics of the fibers to be used for LCB/MOS and SCB are slightly different. In the case of the LCB and MOS the fiber selected is the one from Polymicro FBP $100 / 140 / 170 ; 100 \mu \mathrm{m}$ is the core, $140 \mu \mathrm{m}$ is the cladding and $170 \mu \mathrm{m}$ is the mechanical coating. This fiber has a numerical aperture of $0.2 \pm 0.02$ (optical angle acceptance and output light angle of the fiber, sin (12.71)). This is a wide broadband fiber and provides a good FRD. For the SCB fibers we will use Polymicro fibers with a $70 \mu \mathrm{m}$ silica core and cladding and coating diameters of 140 and $170 \mu \mathrm{m}$, respectively (more details are provided elsewhere in this volume ${ }^{2}$ ).

The estimated length of the fiber link is less than 40 meters. The MEGARA optical fibers for all three instrument modes will be integrated in sub-units of 7 fibers. Each, the LCB sub-units (plus the 8 sub-units coming from the sky positioners that shall be also attached the LCB pseudo-slit), the MOS sub-units, and the SCB sub-units shall be independently covered by two polyurethane loose tubing (not a tight jacket) with an external diameter lower than $40 \mathrm{~mm}$.

The fibers at the fiber cable output will be arranged in a pseudo-slit configuration and position in front of each spectrograph at the pseudo-slit position, one pseudo-slit per instrument mode (see Section 6.1).

\section{MEGARA SPECTROGRAPH}

In Figure 5 we show the 3D view of the detailed design of the MEGARA spectrograph. The positions of the pseudo-slits, the collimator, the VPH in use (located at the instrument pupil), the camera and the cryostat can be easily identified. A major fraction of the area of the MEGARA spectrograph $3 \mathrm{~m} \times 2 \mathrm{~m}$ optical table is occupied by the VPH wheel, where are total of 11 VPHs can be placed.

\subsection{Pseudo-slits}

The optical fibers are mounted on three identical frames (one for each of the pseudo-slits of the three instrument modes, namely LCB, MOS and SCB) that follow the curvature of the entrance focal plane (ROC $=1075 \mathrm{~mm}$ ) for a total length (tangential to the curve) of $119 \mathrm{~mm}$. In order to follow this curvature each frame is split in flat sub-frames (where fibers are attached), called boxes. Each box (of each pseudo-slit) has an integer number of 7-fiber sub-units. The fiber pitch between adjacent fibers within each box is $170 \mu \mathrm{m}$ and their optical axes are all parallel since the system is telecentric.

The pseudo-slits will be moved using two translation stages mounted on $x-y$ that will allow exchanging the pseudo-slit in use between that of LCB, SCB or MOS modes, and also will be used as a focusing mechanism that will be configured in the $\mathrm{z}$-axis for each of these modes and VPH. 


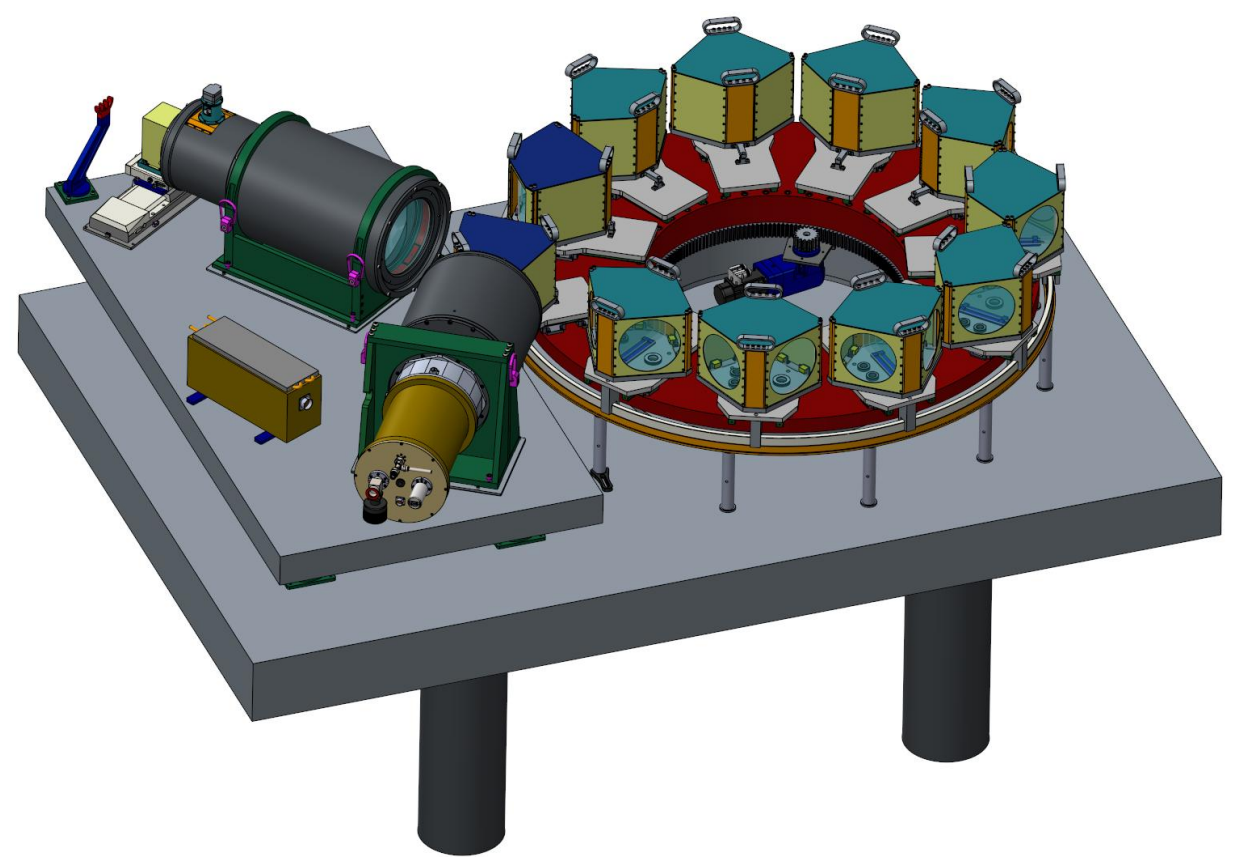

Figure 5. MEGARA Spectrograph Mechanics: 3D view. Here we show the MEGARA spectrograph in one of the two configurations proposed $(\mathrm{MR}+\mathrm{HR})$. The other configuration, $\mathrm{LR}+\mathrm{HR}$, would include all $6 \mathrm{LR}$ VPHs simultaneously mounted on the VPH wheel and as many MR VPHs as possible.

\subsection{Collimator}

The collimator is $\mathrm{f} / 3$ and has a focal length of $484.4 \mathrm{~mm}$ for a total of 5 lenses ( 1 singlet and two doublets). The first lens of the collimator is the only aspherical surface of the instrument, which also one of the smallest lenses in the system $(140 \mathrm{~mm}$ diameter; blank diameter $160 \mathrm{~mm})$. A slit shutter is placed right beyond the first collimator lens. The shutter has three positions: open, closed and filter, position where the order-sorting (OS) filter is placed in the optical path. This latter position will be selected to reject the blue end of the spectrum during the observation with the reddest disperser elements $(>7000 \AA)$. In Figure 6 (left panel) we show the optical design of the spectrograph collimator. All collimator blanks have been already procured and are being polished at INAOE (see right panel of Figure 6).
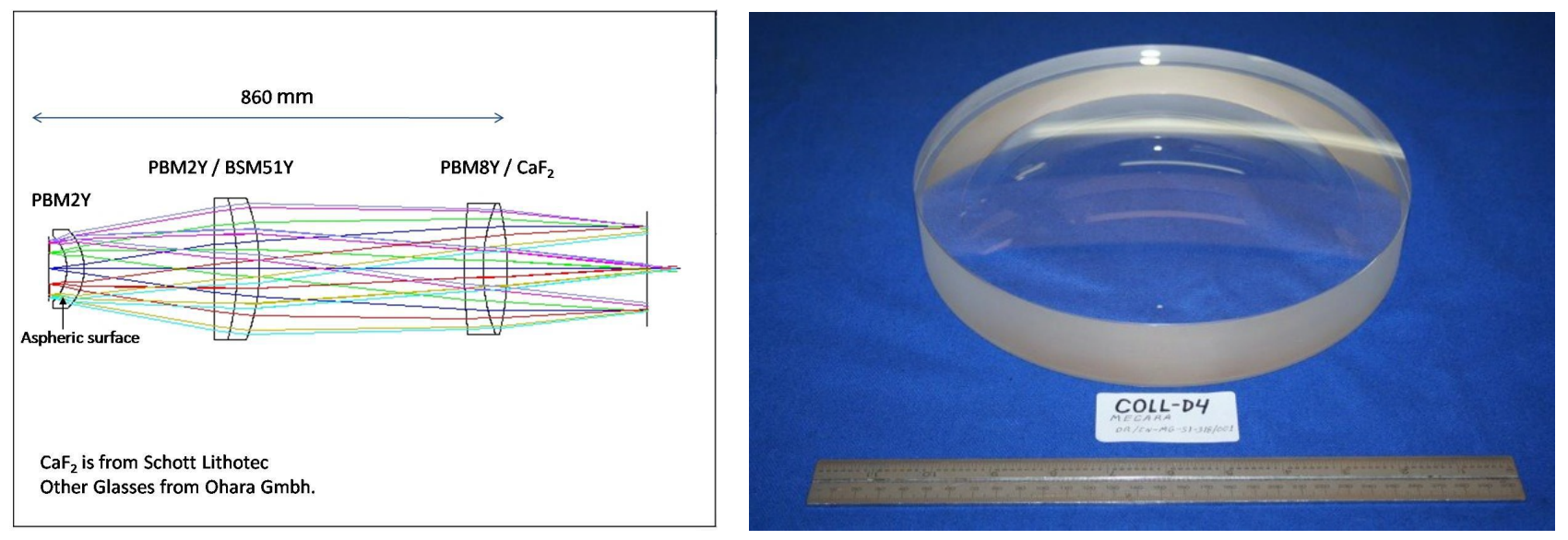

Figure 6. Left: Optical design of the MEGARA collimator. Right: Coll-D4 lens after the polishing process at CIO (more details are given elsewhere in this volume ${ }^{6}$ ). 

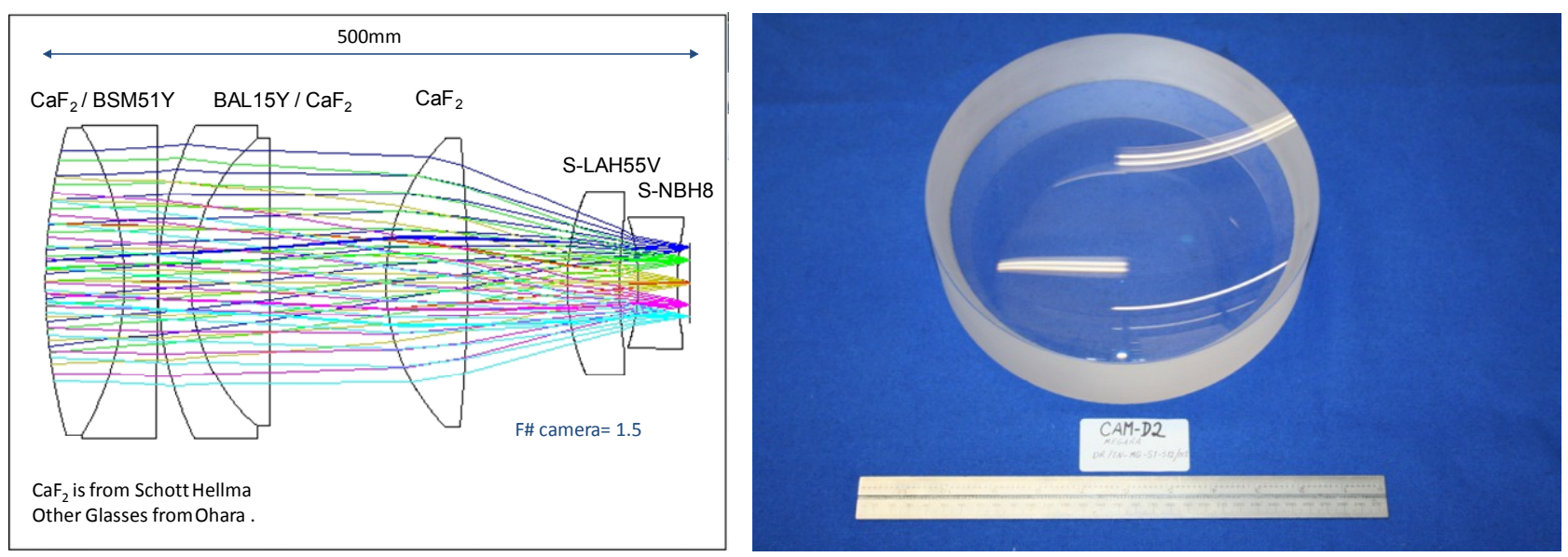

Figure 7. Left: Optical design of the MEGARA camera. Right: Cam-D2 lens after the polishing process at CIO (more details are given elsewhere in this volume ${ }^{6}$ ).

\subsection{Camera}

The camera of the MEGARA spectrograph is an $\mathrm{f} / 1.5$ system with a focal length of $245.9 \mathrm{~mm}$. Two doublets and 3 singlets compose the camera as can be seen in Figure 7. Image field is $61.4 \mathrm{~mm} \times 61.4 \mathrm{~mm}$ that covers the $4 \mathrm{k} \times 4 \mathrm{k} 15-\mu \mathrm{m}$ pixels of the E2V231-84 CCD. The last lens also acts as window for the cryostat. As shown in Figure 5, the angle between the collimator and the camera is fixed to $68^{\circ}$. A detailed view of the a-thermal camera opto-mechanics (along with its interface to the cryostat) is shown in Figure $8^{4}$. Details on the optical design are given in other publications ${ }^{1,13}$. All camera blanks have been already procured and are being polished at INAOE (see right panel of Figure 7).

Table 1. MEGARA VPHs. The resolution, $\mathrm{R}_{\mathrm{FWHM}}=\lambda / \Delta \lambda_{\mathrm{FWHM}}$, is derived from the FWHM $\left(\Delta \lambda_{\mathrm{FWHM}}\right)$ of the $1 \mathrm{D}$ spectra and the case of the LCB IFU and MOS modes (see also Figure 9).

\begin{tabular}{|c|c|c|c|c|c|c|c|c|}
\hline & VPH Name & Setup & $R_{\text {FWHM }}$ & $\begin{array}{c}\lambda_{1}-\lambda_{2} \\
\AA\end{array}$ & $\begin{array}{c}\lambda \mathrm{c} \\
\AA\end{array}$ & $\begin{array}{c}\Delta \lambda(@ \mathrm{c}) \\
\AA\end{array}$ & $\begin{array}{c}\Delta \mathrm{v} \\
\mathrm{km} / \mathrm{s}\end{array}$ & $\begin{array}{c}\text { lin res } \\
\AA / \text { pix }\end{array}$ \\
\hline 1 & VPH405-LR & LR-U & 6028 & $3653-4386$ & 4051 & 0.672 & 50 & 0.17 \\
\hline 2 & VPH480-LR & LR-B & 6059 & $4332-5196$ & 4800 & 0.792 & 49 & 0.20 \\
\hline 3 & VPH570-LR & LR-V & 6080 & $5143-6164$ & 5695 & 0.937 & 49 & 0.23 \\
\hline 4 & VPH675-LR & LR-R & 6099 & $6094-7300$ & 6747 & 1.106 & 49 & 0.28 \\
\hline 5 & VPH799-LR & LR-I & 6110 & $7220-8646$ & 7991 & 1.308 & 49 & 0.33 \\
\hline 6 & VPH890-LR & LR-Z & 6117 & $8043-9630$ & 8900 & 1.455 & 49 & 0.36 \\
\hline 7 & VPH410-MR & MR-U & 12602 & $3917-4277$ & 4104 & 0.326 & 24 & 0.08 \\
\hline 8 & VPH443-MR & MR-UB & 12370 & $4225-4621$ & 4431 & 0.358 & 24 & 0.09 \\
\hline 9 & VPH481-MR & MR-B & 12178 & $4586-5024$ & 4814 & 0.395 & 25 & 0.10 \\
\hline 10 & VPH521-MR & MR-G & 12035 & $4963-5443$ & 5213 & 0.433 & 25 & 0.11 \\
\hline 11 & VPH567-MR & MR-V & 11916 & $5393-5919$ & 5667 & 0.476 & 25 & 0.11 \\
\hline 12 & VPH617-MR & MR-VR & 11825 & $5869-6447$ & 6170 & 0.522 & 25 & 0.13 \\
\hline 13 & VPH656-MR & MR-R & 11768 & $6241-6859$ & 6563 & 0.558 & 25 & 0.14 \\
\hline 14 & VPH712-MR & MR-RI & 11707 & $6764-7437$ & 7115 & 0.608 & 26 & 0.15 \\
\hline 15 & VPH777-MR & MR-I & 11654 & $7382-8120$ & 7767 & 0.666 & 26 & 0.17 \\
\hline 16 & VPH926-MR & MR-Z & 11638 & $8800-9686$ & 9262 & 0.796 & 26 & 0.20 \\
\hline 17 & VPH665-HR & HR-R & 18700 & $6445-6837$ & 6646 & 0.355 & 16 & 0.09 \\
\hline 18 & VPH863-HR & HR-I & 18701 & $8372-8882$ & 8634 & 0.462 & 16 & 0.12 \\
\hline
\end{tabular}




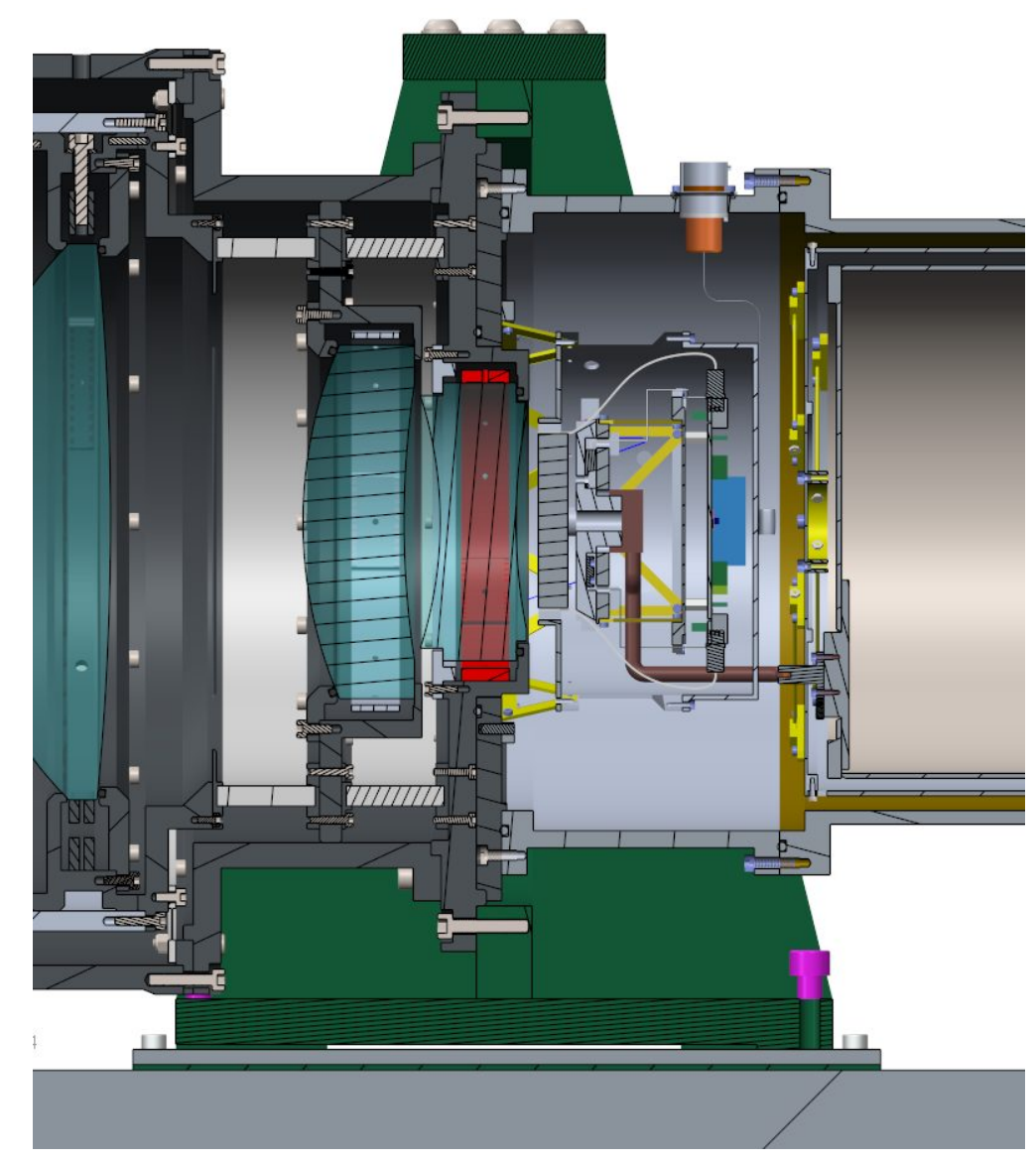

Figure 8. MEGARA spectrograph detailed design. A sectioned view of the camera-cryostat interface and support is shown. See more details elsewhere in this volume $e^{4,5}$.

\subsection{VPHs}

The pupil, which is the location of the VPH gratings, has a $160 \mathrm{~mm}$ free diameter. Different types of pupil elements (all of them based on VPH-type gratings) can be accommodated in the pupil position. The spectral resolutions of MEGARA in terms of $R_{\text {EED80 }}\left(\lambda / \Delta \lambda_{\text {EED80 }}\right)$ are 5,500, 10,000 and 17,000 (respectively for LR, MR and HR VPHs) in the case of the LCB and MOS. When expressed in terms of the FWHM of unresolved lines in the extracted (1D) spectra the spectral resolution becomes $\mathrm{R}_{\mathrm{FWHM}} \sim 6,000$ (low resolution; $\mathrm{LR}$ ), $\mathrm{R}_{\mathrm{FWHM}} \sim 12,000$ (mid-resolution; MR) and $\mathrm{R}_{\mathrm{FWHM}} \sim 18,700$ (high resolution; HR). With regard to the $\mathrm{SCB}$ the spectral resolutions reached are $\mathrm{R}_{\mathrm{FWHM}} \sim 7,000,13,500$ and 21,500 respectively for LR, MR and HR VPHs. With regard to the wavelength coverage MEGARA offers full optical coverage with the LR VPHs $\left(\mathrm{R}_{\mathrm{FWHM}} \sim 6,000\right)$ alone or with the MR VPHs $\left(\mathrm{R}_{\mathrm{FWHM}} \sim 12,000\right)$ combined. In Figure 8 we show the coverage in terms of wavelength and spectral resolution of the different spectral setups available in MEGARA. This figure corresponds to the performance provided by the LCB and MOS. In the case of the SCB the spectral ranges are identical but spectral resolutions are typically higher by $15 \%$.

In the case of the highest resolution mode (HR VPHs), the incident angles are so high that the use of single monolithic prisms implies very large prisms and a small amount of vignetting of $\sim 10-19 \%$, depending on wavelength. In the case of the MR VPHs we found that the standard Kogenik method was yielding very low efficiencies in one of the polarizations, so we decided to use the HD manufacturing technology patented by Wasatch Photonics instead, as this allowed recovering rather flat and high efficiency curves in both polarizations. In addition to the broad-band coatings of the collimator and camera lenses the Centro de Investigaciones en Óptica (CIO, Mexico) has also designed specific narrowband coatings for the VPHs windows and prisms. 


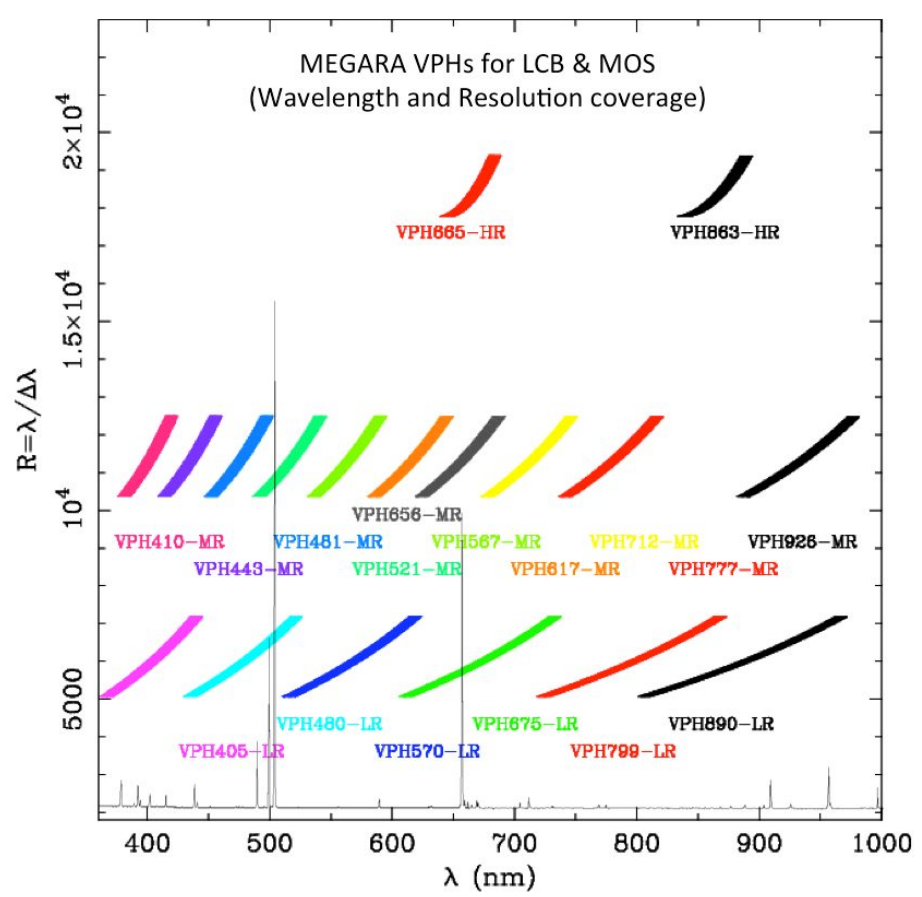

Figure 9. Coverage of the MEGARA VPHs in resolving power $\left(\mathrm{R}_{\mathrm{FWHM}}\right)$ and wavelength for the LCB IFU and MOS modes (100 $\mu \mathrm{m}$-core fibers). Note that, due to the use of VPHs, the spectral resolution changes across the detector, which spans an angle of $\pm 7.2^{\circ}$ from the pupil, although this variation is always within $\pm 20 \%$.

\section{CRYOSTAT}

The selected cryogenic device to harbor the CCD detector for the MEGARA spectrograph is a liquid nitrogen open-cycle cryostat. This LN2 open-cycle cryostat is a custom made product, which has been designed by the INAOE astronomical instrumentation group. The proposed cryostat offers modular stages for easy assembly and testing whilst also allowing future modifications to accommodate the required CCDs, electronics and optics. Cryostat mounting is horizontal and it is designed to be kept static, as well as the other MEGARA spectrograph components. The complete cryostat assembly consists of two main parts: the dewar back and the CCD Head (see left and right panels of Figure 10, respectively).

The dewar back (or main body) serves as vacuum jacket and contains the liquid nitrogen tank; it also has on the rear part the liquid nitrogen fill tube, an electrical port for temperature monitor and two vacuum ports. Aluminum has been selected as the primary material for the vacuum jackets described in this document; aluminum alloys offer a good structural choice, easy manufacturability (compared with stainless steel), reduce costs of fabrication and weight but it also offers a very low degassing rate compared to stainless steel for untreated materials and the hydrogen permeation rate is also lower than steel. LN2 tank will be made of stainless steel (which has low thermal conductivity). The filling tube has a bellow system, which helps to reduce thermal loading on the cryogens. Cold plate will be made of gold plated OFHC copper to increase thermal conductivity.

The CCD Head is assembled on top of the main body and will contain the CCD detector and its associated electronics; it will contain two electrical ports to read-out the signals from the detector. CCD supports will be made of low thermal conductivity materials (G10, nylon or Teflon). The CCD detector will be thermally connected to the LN2 tank through a high purity free oxygen copper strap, which can be adjusted to give the desired operating temperature for the detector. A lid on the front part will contain the last lens of the MEGARA spectrograph, which will also serve as a vacuum window. The CCD head mechanical module can be disassembled completely from the cryostat main body for easy handling and integration and verification of CCD components. An aluminum radiation shield that will help to improve the hold-time of the cryostat will surround the CCD and its mountings.

More information of the detailed design of the MEGARA cryostat is given elsewhere in this volume ${ }^{5}$. 

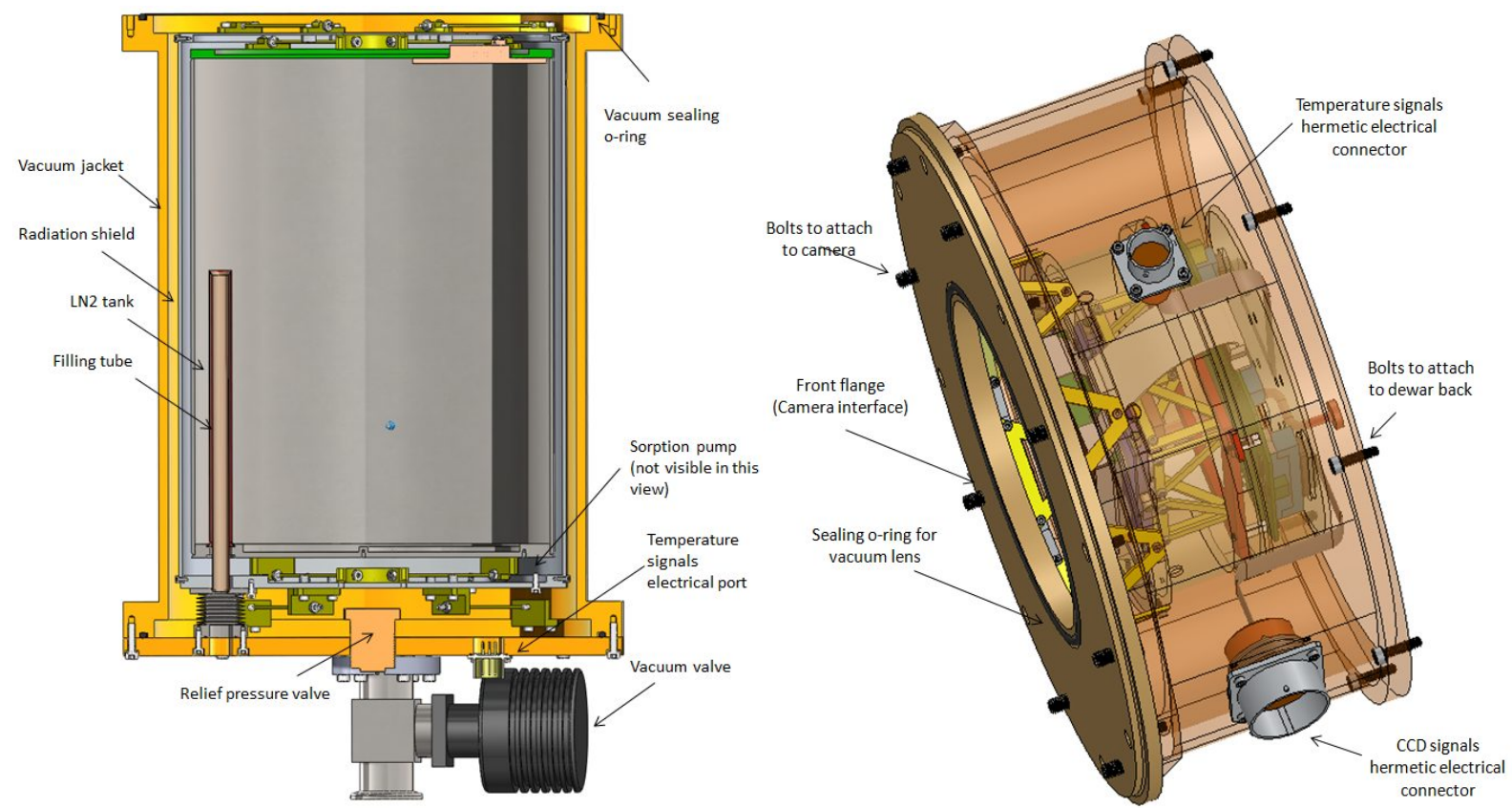

Figure 10. Left: Cryostat dewar back view. Right: CCD Head.

\section{CCD DETECTOR}

The MEGARA detector will be an E2V CCD231-84-0-E74 device. This measures 4096 x 4112 x $15 \mu \mathrm{m}$ pixels and has four outputs. There are several variants of this device but we have chosen the Deep-Depletion Silicon version with the Astro Multi-2 AR coating as being the one best suited for the demands of MEGARA. This CCD is capable of delivering $<3$ e- read noise and has excellent QE across almost the whole visible spectrum. In Figure 11 we show the overall system efficiency for this CCD and considering the different VPHs to be used with MEGARA.

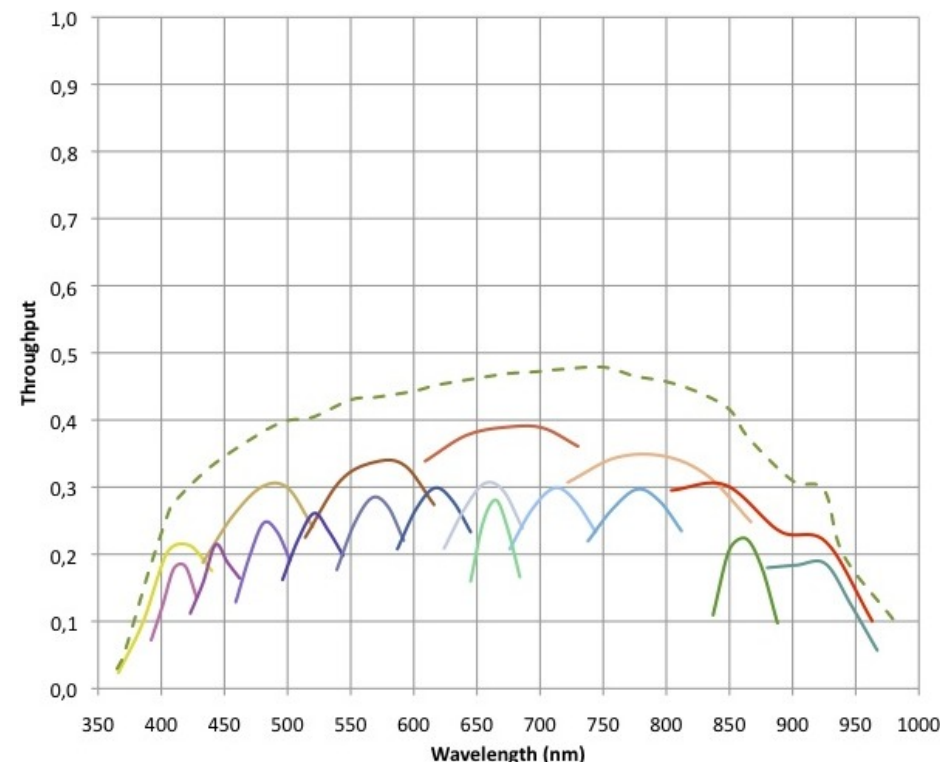

\section{MEGARA LCB/MOS}

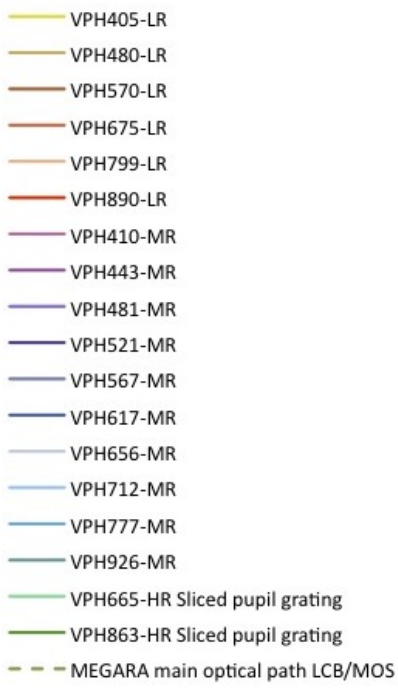

Figure 11. System overall efficiency with no VPH and for the different VPHs designed for its use with MEGARA. 
The use of Deep Depletion Silicon means that fringing in the red part of the spectrum is minimized. The CCD will be mounted in an LN2 cryostat and operated at approximately $158 \mathrm{~K}$ to ensure low-noise operation. The positioning of the CCD within the cryostat is critical since the cryostat window will be active. The assembly of the camera will be greatly facilitated by the CCD package, which is designed for mosaicking applications. It has a thickness of $15 \pm 0.01 \mathrm{~mm}$ and contains three mounting bolts to ensure tight thermal contact with the base plate. Figure 8 shows a lateral view of the CCD and PCB at the CCD head.

\section{MEGARA CONTROL SYSTEM}

The MEGARA Control System (MCS) includes the hardware and software components that are required to provide the MEGARA control and the integration of the instrument in the GTC Control System (GCS). Additionally, the MEGARA project includes the design and development of the software tools that shall be developed by the MEGARA Consortium to facilitate the preparation of the observing programs and the exploitation of the data provided by the instrument to the GTC community, including the MEGARA Observing Preparation Software Suite (MOPSS), which is composed by the Exposure Time Calculator (ETC), the Simulator and the Fiber MOS Positioning Tool (FMPT), the Off-line MEGARA Data Reduction Pipeline and the On-line Pipeline.

\subsection{MCS Software}

MEGARA Control System will provide the capabilities to move the different mechanisms of the instrument, to readout the data from the detector controller, the necessary routines for the Inspector Panels and the Sequencer strategies.

The MEGARA Mechanisms Control System shall be composed by a set of devices that represents the different MEGARA components. These components (i.e., focal-plane cover, shutter, focusing, grating exchange mechanisms, Fiber MOS positioners, detector, etc.) shall receive the positioning demands requested by the user in the Observing blocks and provide the data, monitors and alarms that must be generated to the GCS.

\subsection{Science Community tools}

MEGARA Science Community Tools are stand-alone applications that are developed by the MEGARA Consortium to facilitate the preparation of the observing programs and the reduction of the data obtained with the instrument.

- The MEGARA Observing Preparation Software Tools (MOPSS) shall assist observers to optimally plan their observations. The MOPSS is composed by the Exposure Time Calculator, the Image Simulator, and the FiberMOS Positioning tool.

- The MEGARA Exposure Time Calculator shall simulate the signal-to-noise $(\mathrm{S} / \mathrm{N})$ ratios that will be obtained for the continuum and a spectral line of a target for a given exposure time, MEGARA setup, and night atmospheric conditions.

- The MEGARA Image Simulator shall simulate data frames of any idealized distribution of sources in the sky in the same way it they would have been observed by MEGARA for a given setup. The Simulator shall return a MEGARA frame in FITS format with the simulated spectra corresponding to the projection of each spaxel on the detector plane, including the expected sky contribution and the effects inherent to the observation (bias, flat, geometrical distortion, non-linear dispersion, crosstalk, differential atmospheric refraction), as well as its row stacked spectra frame.

- The Fiber MOS Positioning tool shall determine the optimal assignment of the 92 positioners used in the MOS mode for an input list of source coordinates in the $3.5 \times 3.5 \operatorname{arcmin}^{2}$ to cover as many sources as possible, and provide in which order must be the positioners be moved to avoid collisions among adjacent ones (minimizing the time to configure them at the same time).

- The MEGARA Data Reduction Pipeline (MDRP) shall supply the users with data corrected from instrument signatures, which can be used at different stages of data acquisition and analysis. The goal of the MDRP is to supply users with a final data set in physical units, with which they can begin their scientific analysis, without the need of additional data processing. The user shall be able to modify the predefined parameters of the MDRP to customize data processing. The On-line MEGARA pipeline routines shall be provide part of the off-line functionality (MEGARA shall provide these routines to GTC to facilitate the GTC control team the development of this application). 


\subsection{MCS Hardware}

The MEGARA control system hardware will be divided into two separated parts (physically or logically depending on the final electronic cabinets characteristics). The Control cabinet will gather all the workstation and interface to the GTC control system. The Power Cabinet will gather all the power electronic, mainly DC motor drivers and power supplies. Both cabinets will be equipped with an $\mathrm{AC}$ panel that provides a filtered $230 \mathrm{~V}$ AC to the cabinets (see Figure 12).

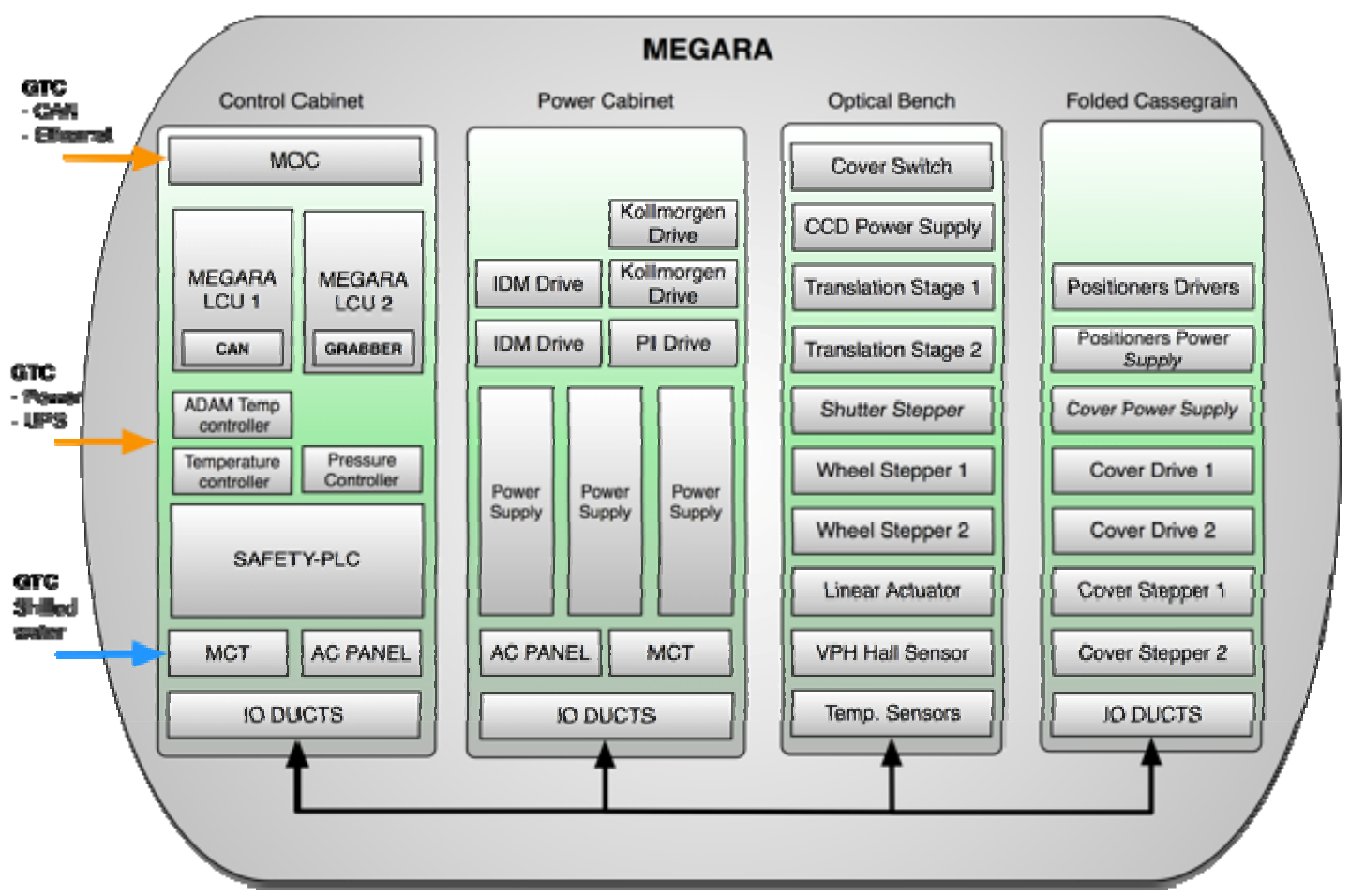

Figure 12. MEGARA Control System hardware overview.

\section{SYSTEM ENGINEERING AND MANAGEMENT PLAN}

The main tasks that are carried out as part of the Systems Engineering activities within the MEGARA project are:

- Implementing the requirements engineering, which aims to: ensure that the initial requirements are correctly interpreting user needs; generate, monitor and maintain a coherent set of specifications at different levels of the system and ensure traceability between subsystem and system requirements and specifications.

- Performing system analysis, resolve requirement conflicts, carry out trade-off, develop and use simulation models, analyze project risks and perform RAMS analysis.

- Defining and maintaining system configuration (define Product Tree and Interface Table).

- Preparing and executing the Integration and Verification Plan.

- Developing the Operation and Maintenance Plan.

This System Engineering Plan was developed for the complete system life cycle, from conceptual design to the final instrument acceptance in GTC. The activities must be reviewed at the end of each phase in order to add the needed details to the activities (WBS) to be performed in the following phase. The System Engineering activities for the Detailed Design have been reviewed at the end of the Preliminary Design Phase ${ }^{3}$. 
The MEGARA Management Plan at Detailed Design level is aimed at fitting all the managerial parameters listed in the following subsections. This Management Plan is based on the contracts for the detailed design, construction, assembly, integration, verification (AIV) and commissioning of MEGARA signed on April $28^{\text {th }}$ and May $5^{\text {th }} 2014$ between GRANTECAN S.A. and the UCM ${ }^{7}$.

\subsection{Leadership}

The UCM, as leader institution and responsible for many MEGARA work packages, is committing their best resources both scientifically, managerial and engineering, and also putting the needed energy to develop MEGARA and making MEGARA to become a workhorse instrument for the GTC and a reference instrument in the large telescope league. UCM also commits to give the PI and the team the needed support and stability to be able to do their work over the project life.

The MEGARA Consortium, formed by UCM, INAOE, IAA and UPM, has been firmly established with a Letter of Agreement and Commitment (LoAC) already signed back in 2011 by the representatives from each institution. This LoAC has been recently updated (June 2014) on the view of the final construction contract signed with GRANTECAN S.A. All partners have given MEGARA project the highest priority and have devoted the best resources and facilities for its development.

\subsection{Scope}

MEGARA-as-contracted has a MOS mode with 92 positioners, plus 8 fixed positioners for sky subtraction fitted in the same pseudo-slit that the LCB fibers. The LCB and MOS modes cannot operate simultaneously since MEGARA has one single spectrograph, although the exchange is very fast (seconds) since all the bundles are mounted simultaneously on the instrument, sharing the same mechanism. MEGARA shall be equipped with 18 pupil elements (6 LR, 10 MR and 2 HR), 11 of them simultaneously mounted on the spectrograph.

The SCB mode has no funds assigned in the framework of the present contract with GRANTECAN but MEGARA Consortium is actively looking for those funds. The design is fully finished and all provisions have been made in the instrument so the ordering can take place at the beginning of 2015, on time for its integration with MEGARA at UCM.

MEGARA has been detailed designed with no major risks either shop-stoppers. We have not identified any subsystem that has major issues from the technical point of view. We have analyzed and taken into account the comments given by the external panels and by GRANTECAN at the time of the previous reviews and the current design and project plan complies with all requirements both from GRANTECAN by contract and MEGARA Science Team, providing a solid solution for the instrument design and development. MEGARA has a robust and convincing instrument design with a high versatility and complete set of observing modes with an excellent efficiency/cost rate.

\subsection{Schedule}

The delivery time of all subsystems is well known and we can calculate the schedule quite precisely. Current date is compatible with the contractual dates: a laboratory acceptance in February 2016, a delivery to the observatory in November 2016 (or before) and a commissioning finished before the end of April 2017.

\subsection{Budget}

This is a co-funded project by GRANTECAN and by MEGARA Consortium. The total project budget adding the money with the in-kind contribution converted in $€$ is $11.8 \mathrm{M} €$, being 6.5 million euros in money, including a contingency of $\sim 0.43 \mathrm{M} €$. The rest is in-kind contribution of the Consortium ( $\sim 5.3 \mathrm{M} €)$. This amount corresponds to $45 \%$ of the total project budget. Should we convert this money in Guaranteed Time (GT) according to GRANTECAN conversion (220 hours per million euros), the equivalent GT would be 1166 hours. Despite the Announcement of Opportunity issued by GRANTECAN on 2009 offered an envelope of 660 hours (well below the 1166 hours quoted above), the GT finally awarded was only 301 hours.

The major project driver that has determined (and will do) the project parameters and in particular the schedule and consequently the overall budget is an appropriate cash flow. We have designed a plan to fit the cash flow agreed in the contract according to the planned the project expenses. 


\section{REFERENCES}

[1] Ortiz, R., et al., "Inverse analysis method to optimize the optic tolerances of MEGARA: the future IFU and Multi-Object Spectrograph for GTC", Proc. SPIE, this volume (2014)

[2] Pérez-Calpena, A., et al., "MEGARA fiber bundles", Proc. SPIE, this volume (2014a)

[3] Pérez-Calpena, A., et al., "System engineering at the MEGARA project", Proc. SPIE, this volume (2014b)

[4] Castillo, E., et al., "MEGARA main optics opto-mechanics", Proc. SPIE, this volume (2014)

[5] Ferrusca, D., et al., "MEGARA cryostat advanced design", Proc. SPIE, this volume (2014)

[6] Carrasco, E., et al., "MEGARA Optical manufacturing process", Proc. SPIE, this volume (2014)

[7] García-Vargas, M.L., et al., "Project management for complex ground-based instruments: MEGARA plan", Proc. SPIE, this volume (2014)

[8] Castillo-Morales, A., et al., "MEGARA Control System", Revista Mexicana de Astronomía y Astrofísica (Serie de Conferencias) 42, 123 (2013)

[9] Sheth, K., et al., "The Spitzer Survey of Stellar Structure in Galaxies $\left(\mathrm{S}^{4} \mathrm{G}\right)$ ", Publications of the Astronomical Society of the Pacific 122, 1397-1414 (2010)

[10] Egami, E., et al., "The Herschel Lensing Survey (HLS): Overview", Astronomy \& Astrophysics 518, L12, 5 pp. (2010)

[11] Gil de Paz, A., "MEGARA: the future optical IFU and multi-object spectrograph for the $10.4 \mathrm{~m}$ GTC telescope", Proc. of SPIE Vol. 8446, 84464Q (2012)

[12] Gil de Paz, A., et al., "MEGARA: The future IFU and MOS of the $10.4 \mathrm{~m}$ GTC", Revista Mexicana de Astronomía y Astrofísica (Serie de Conferencias) 42, 90-92 (2013)

[13] Carrasco, E., et al., "MEGARA spectrograph optics", Proc. of SPIE Vol. 8011, 80112D (2012) 